\title{
Características Sociodemográficas de las personas con conducta acumuladora/trastorno por acumulación (S. de Diógenes) en la ciudad de Madrid. Serie de casos.
}

Sociodemographic characteristics of persons with hoarding behavior/hoarding disorder (Diogenes syndrome) in the city of Madrid. Cases study.

\author{
Emiliano Rodríguez Lozano a , Carmen Ortiz Fuillerat ${ }^{\mathrm{b}}$, Gema Blasco Novaldos ${ }^{\mathrm{c}}$, Mercedes Sáez \\ Antón d, Fernando García Gutiérrez ${ }^{\text {e }}$, Carmen Bermejo Pérez ${ }^{\text {f }}$. \\ ${ }_{a, b, c, d, e y f}$ Madrid Salud. Departamento de Salud Ambiental. Unidad Técnica de Entorno Urbano y Vivienda. \\ Madrid, España.
}

Correspondencia: Emiliano Rodríguez Lozano (rodriguezle@madrid.es)

Recibido: 03/01/20143; aceptado: 06/04/2014

\begin{abstract}
RESUMEN: Motivos. El trastorno de conducta por acumulación origina significativos riesgos para la salud del acumulador y para la Salud Pública, problemas de convivencia en el entorno familiar y vecinal y amenazas para la seguridad motivada por el riesgo de incendios. Finalidad. El presente estudio tiene como objetivo describir las características sociodemográficas de los acumuladores en la ciudad de Madrid.

Método. Fueron seleccionados 295 casos de las 1147 solicitudes de intervención por posibles situaciones de insalubridad que entraron desde el 1 de enero de 2009 al 31 de diciembre de 2012 a la Unidad Técnica de Entorno Urbano y Vivienda de Madrid Salud. Los casos cumplían todos o alguno de los criterios diagnósticos del acumulador patológico descritos por Randy $\mathrm{O}$ Frost et al en 1993(1).

Datos. Los acumuladores tienen una edad media de 64.77 años, son hombres en el $55.9 \%$ de los casos y el $47 \%$ son mayores de 65 años. Son españoles el $95.6 \%$, y pensionistas $65.1 \%$. En 129 casos se retiraron un total de 260.346 kgrs de basura y enseres.

Conclusiones. El trastorno por acumulación, aunque poco frecuente, provoca un grave problema a la persona que lo sufre, un riesgo para la Salud Pública y para la seguridad y un alto coste por la elevada cantidad de recursos que consume su atención y resolución.

Nuestro trabajo añade argumentos para considerar el trastorno de acumulación como una entidad independiente como ya se clasifica en la DSM-5 de mayo de 2013.

PALABRAS CLAVE: Características Demográficas. Conducta acumuladora. Trastorno por acumulación. Síndrome de Diógenes. Acumulador de animales. Comorbilidad. Prevalencia.
\end{abstract}

ABSTRACT: Background. Hoarding disorder causes significant health risks for hoarder and to Public Health, family and neighbouring and security threats motivated by fire hazard.

Objective. The present study aims to describe the sociodemographic characteristics of hoarders in the city of Madrid.

Method. Setting. 295 cases were selected from 1147 applications for assistance for any unhealthy situations that went from 1 January 2009 to 31 December 2012 at the Technical Unit of Urban Environment and Housing of Madrid Salud. Cases meet any or all of the diagnostic criteria for pathological hoarder described by Randy O Frost et al in 1993. Results. Hoarders have an average age of 64.77 years, are men in $55.9 \%$ of cases and $47 \%$ are over 65 years. The majority, $95.6 \%$ are Spanish, and are pensioners $65.1 \%$. In 129 cases a total of 260,346 $\mathrm{kgs}$ of trash and belongings were removed.

Conclusions. Hoarding disorder, although rare, causes a serious problem for the person who suffers, a risk to Public Health and safety and a high cost for the high amount of resources his attention and resolution consumes.

Our work adds arguments to consider the hoarding disorder as an independent entity like is classified in the DSM-5 of may 2013.

KEY WORDS: Demographics characteristics. Hoarding behaviors. Hoarding disorder. Diogenes Syndrome. Animal hoarding. Comorbidity. Prevalence. 
ORIGINALES Y REVISIONES

\section{Introducción:}

Como ocurre con la mayoría de las conductas humanas, el hecho de guardar y coleccionar posesiones puede variar desde normal y adaptativa hasta excesiva o patológica (2-3). No es hasta principios de la década de los 90 cuando aparece el primer amplio estudio relativo a la conducta acumuladora por_Randy O Frost, Gail Sketekee, David F Tolin and Tamara L Hartl (4). La conducta acumuladora se define entonces como un fenómeno complejo y es considerada como un trastorno cuando la excesiva acumulación de objetos, enseres, basura o su incapacidad para eliminar lo considerado inútil o aparentemente sin valor, interfiere en el desarrollo normal (personal, profesional, económico, familiar y social) de la persona afecta, causándole un significativo estrés (1, 5-6). Una definición ampliamente aceptada se concreta en una conducta inadecuada de acaparar y guardar y la dificultad de deshacerse de objetos de escaso valor originando acumulación y desorden, desasosiego y discapacidad (1). El grado del desorden y la magnitud de la ocupación de los espacios habitables es el factor que más claramente pone de manifiesto el grado de deterioro de estas personas (7).

Hay autores que clasifican la conducta acumuladora en 8 tipos diferentes (8-9).

El más conocido por mediático, pero no el más frecuente, sería el mal llamado Síndrome de Diógenes (10) referido a aquellos casos de personas con trastorno de conducta mayores de 65 años, en aislamiento social, acumuladores de objetos inservibles y/o basura orgánica, abandono de sí mismo (autonegligentes), rechazo de la ayuda externa y nula conciencia de la anomalía de conducta. Otro caso especial, extremo e infrecuente, es el Síndrome de Noé, definido como el trastorno de conducta que acumula animales domésticos, principalmente perros y gatos, en el domicilio propio junto con el intento obsesivo de mantener e incrementar su número, acompañado del fracaso de mantener el mínimo cuidado de los mismos y de la nula conciencia de la anomalía de esta conducta y de las consecuencias para los animales(desnutrición, enfermedad y muerte); comparte con el Diógenes el aislamiento social, el rechazo de la ayuda externa y la hostilidad ante esta ayuda.

Los factores que hacen clínicamente visibles a los acumuladores son el estrés y la interferencia con sus actividades normales.

Acumulador o Trastorno por Acumulación son los términos empleados para referirnos a esta forma excesiva (11). La ausencia de una definición clara y la imposibilidad de extraer una muestra representativa hace que en la actualidad la única posibilidad de acercarnos a este problema sea a través de series de casos.

Crecientes evidencias epidemiológicas, fenomenológicas, neurobiológicas y estudios de tratamientos, sugieren que se trata de un síndrome autónomo con sus propios criterios diagnósticos y no un síntoma de otra enfermedad (2, 11-12).

Hasta el año 2012 era incluido en el DSM-4 (Diagnostic and Statistical Manual of Mental Disorder; American Psychiatric Association, 1994) como un sínto- 
ORIGINALES Y REVISIONES

ma más del Trastorno Obsesivo Compulsivo; en la actualidad ha pasado a constituirse como una entidad independiente denominada"Hoarding Disorder" (trastorno por acumulación) en el DSM-5 (American Psychiatric Association, 2013); la ICD10 (International Classification of Diseases) no incluye este trastorno $(2,13)$.

La persona que acumula no es consciente, la mayoría de las veces, de la gravedad de su comportamiento. Lo consideran normal y necesario y oponen gran resistencia a cualquier intento de ayuda (14). Sin embargo se trata de un trastorno crónico y debilitante que en los casos severos impide el uso normal de los espacios para desarrollar las actividades básicas como cocinar, limpiar, desplazarse por el interior de la vivienda incluso dormir. Puede convertirse en un grave problema al existir riesgo de incendio (12), caídas, riesgo para la salud individual (infestaciones, desnutrición) y del entorno como un significativo problema para la Salud Pública (14).

La conducta acumuladora es un trastorno psicológico invalidante que ha sido observada en varios trastornos neuropsiquiátricos como demencia, esquizofrenia, trastornos de la alimentación, autismo, depresión y más comúnmente en el trastorno obsesivo-compulsivo, pero también en personas carentes de patología clínica (14). El acumulador no necesariamente es abandonado o con falta de higiene ni presenta patología conocida ni reconocible. Está asociada a una sustancial incapacidad funcional y representa una gran carga para su familia y la sociedad en general $(11,14)$.

La prevalencia estimada en la población a partir, fundamentalmente, de estudios en individuos con trastorno obsesivo-compulsivo, oscila según diferentes estudios de un $0.4 \%(15)$ al $2-5 \%(16-21)$. Siendo esta cifra muy controvertida dada la ausencia de estudios con criterios diagnósticos sistematizados y estandarizados, considerando al acumulador compulsivo como una entidad diferenciada.

Los estudios más recientes muestran que los acumuladores son más prevalentes y suponen y una carga mayor que la que se pensaba.

El desconocimiento que existe en relación con este síndrome hace que incluso algunos expertos pongan en duda su existencia; se trataría de una elección vital, de un estilo de vida (22). Sus límites diagnósticos permanecen aún como materia de debate (11).

En los últimos tiempos se ha incrementado de manera significativa el interés por este trastorno de conducta aunque en España los estudios siguen siendo muy escasos y la base del conocimiento de este problema viene de estudios realizados en otros países.

Necesitamos conocer mejor los componentes de este tipo de trastorno para podernos acercar al mismo desde un punto de vista psicológico y social que nos permita mejorar su abordaje integral.

Hasta la fecha no se ha publicado ningún estudio que evalúe las características sociodemográficas de los acumuladores desde la perspectiva de las consecuencias físicas y sociales que ocasionan la conducta acumuladora. 
El manejo de las denuncias de casos potenciales de acumuladores patológicos en la ciudad de Madrid está exclusivamente canalizado a través de su Ayuntamiento.

Las especiales características que configuran este trastorno, según la bibliografía y la experiencia acumulada, nos hace pensar que la casi totalidad de este tipo de casos en la ciudad de Madrid pasan por nuestra Unidad Técnica.

El presente estudio tiene como objetivo principal conocer las características sociodemográficas de las personas con Trastorno por Acumulación en la ciudad de Madrid que han sido gestionados en la Unidad Técnica de Entorno Urbano y vivienda desde el 1 de enero de 2009 al 31 de diciembre de 2012 y como objetivos secundarios: a) conocer cuántas personas son mayores de 65 años, viven solas y sufren este trastorno de conducta, b)determinar si la conducta acumuladora es más frecuente y difiere entre los hombres y las mujeres, c)estudiar si hay relación entre las características del material acumulado con la edad y el sexo, d)conocer si la nacionalidad influye en la conducta acumuladora, e)estudiar si existe alguna relación entre la conducta acumuladora con el nivel educativo y la situación laboral, f) determinar si el hecho de vivir solo se relaciona con el grado de acumulación, g) conocer cuántos atesoradores de animales (síndrome de Noé) hay, h) estudiar qué patología prevalece en los acumuladores, si ésta es diferente según se trate de hombres o mujeres y si se relaciona con el tipo de acúmulo, i) conocer si los casos resueltos con autorización judicial son más reincidentes que el resto, y finalmente , j) conocer a qué edad son más reincidentes.

\section{Material y Método}

\section{Diseño del Estudio.}

El Ayuntamiento de Madrid, a través del organismo autónomo Madrid Salud, dispone de la Unidad Técnica de Entorno Urbano y Vivienda, adscrita al Departamento de Salud Ambiental , que tiene como funciones, entre otras, dar respuesta a las solicitudes de particulares o de otras instancias administrativas(Comunidades de Vecinos, Servicios Sociales, Policía Municipal, Bomberos, familiares y vecinos en general) que denuncien una situación de insalubridad en viviendas o locales del municipio de Madrid que suponga un riesgo para la salud de los vecinos o para la población. A partir de cada una de las denuncias se abre el correspondiente expediente administrativo; se obtienen hasta un total de 80 variables que se registran en un documento de acces elaborado ex profeso para ello de las que se vuelcan a una hoja de cálculo un total de 67 variables. La información es recogida en el lugar denunciado a partir de lo observado por tres inspectores, siempre los mismos. También se registra la información que se obtiene a través de la familia, vecinos, propietario, Servicios Sociales, Padrón Municipal, Samur Social, Bomberos, Policía Municipal o del propio acumulador. Cuando se ha permitido el libre acceso a la vivienda o si finalmente se ha accedido al domicilio mediante auto- 
rización judicial de entrada en el domicilio para realizar el saneamiento, los inspectores comprueban mediante observación directa, al menos una vez, las características de la vivienda, el estado de insalubridad y habitabilidad de la misma.

Participantes y recolección de datos

Desde el 1 de enero del 2009 al 31 de diciembre de 2012 se recibieron un total de 1147 solicitudes de intervención por posibles casos de insalubridad en viviendas y/o locales sin actividad económica, de los que 295 presentaban el trastorno por acumulación al cumplir todos o algunos de los criterios siguientes: a) excesiva acumulación de objetos, enseres $\mathrm{y} / \mathrm{o}$ basura; $\mathrm{b}$ ) incapacidad para eliminar lo considerado inútil o sin valor; c) interferencia en el desarrollo normal(personal, profesional, económico, familiar y social) de la persona o personas afectas, causándole un significativo estrés y d) imposibilidad de hacer un uso normal de ese espacio para el que estaba diseñado $(1,4-6,19,23)$.

El diagnóstico de trastorno por acumulación se ha hecho a través de las consecuencias físicas en el entorno personal, familiar y vecinal originado por el comportamiento del sujeto acumulador; no se han usado test ni cuestionarios específicos (24) para aproximarnos al diagnóstico clínico de este trastorno de conducta, por lo que no ha sido preciso la firma de consentimiento por parte de los afectados.

Los datos se han obtenido de la revisión de la base de datos de los expedientes, siendo los métodos para la recogida de información la entrevista personal, la observación directa, así como los aportados por otras áreas municipales, otros organismos relacionados, familiares y vecinos.

Variables de estudio

La edad, nacionalidad y nivel de estudios es la registrada en el padrón municipal o la que manifiesta el acumulador en el momento de abrir el expediente. El sexo se recoge por el Padrón Municipal y el que observan los inspectores.

La patología y adicciones se obtiene de los informes clínicos, información de los servicios sociales, familiares, manifestado por el/la afectado/a y evidenciado por los inspectores.

El distrito de residencia se obtiene a través del Padrón Municipal.

La situación laboral es la que manifiesta el afectado a los inspectores. Se ha considerado pensionista a los que tienen una pensión como tal y aquellos que reciben algún ingreso periódico por parte de la administración tipo RMI (renta mínima de inserción de la Comunidad de Madrid).

La tipología y distribución del acúmulo se categoriza según lo que predomina en el material acumulado.

La cantidad de kgs retirados los facilita el Departamento de Explotación de Recogida de Residuos del Ayuntamiento de Madrid que es quien realiza las labores de saneamiento coordinados por la Unidad Técnica de Entorno Urbano y Vivienda.

Reincidentes se considera los casos en que conste en nuestra Unidad uno o más expedientes previos por insalubridad archivados porque se realizó el saneamiento. 
Si es sociable (se relaciona con los demás), reservado (circunspecto, poco hablador) o agresivo (que responde o actúa de forma violenta), en aislamiento social se obtiene de la información de servicios sociales, familiares, vecinos u observado por los inspectores.

La presencia, situación socio-sanitaria y número de animales son referenciados según lo observado por los inspectores, considerándose como Síndrome de Noé cuando coincide tenencia de animales en malas condiciones higiénico-sanitarias.

Análisis de datos

Se trata de un estudio descriptivo de una serie de casos. Para el análisis de los datos se presentan distribuciones de frecuencia para las variables cualitativas y medias y desviación estándar para las variables cuantitativas, así como representación gráfica de los datos. Los datos fueron analizados mediante paquete estadístico SPSS (Statistical Package for de Social Sciencies) versión 15.0 para window.

\section{Resultados:}

\section{Sociodemográficos}

De las 1147 solicitudes de intervención 295 (25.8\%) cumplían los criterios de acumulador patológico.

Los acumuladores tienen una edad media de 64.77 años (DS de 15; rango de 23-96 años), de los que son varones 165 (55.7\%); tienen más de 65 años 139 (47.1\%). Las mujeres acumuladoras son mayores que los hombres, tienen una edad media de 67.24 años (DS: 15) y 62.79 años (DS: 15) respectivamente.

Las que más acumulan son las mujeres mayores de 65 años $68(53.5 \%)$ y tanto en hombres como en mujeres la prevalencia de acumuladores se incrementa con la edad. Son analfabetos $46(18.8 \%)$ y $79(32.2 \%)$ saben leer y escribir.

Viven solos 132 (44.7\%), en aislamiento social 32 (10.9\%); La gran mayoría son pensionistas 149 (65.1\%). Se considera insociables a $204(69.28 \%)$, reservados a $56(19.0 \%)$, agresivo $23(7.8 \%)$. Carecen de familia $252(85.7 \%)$.

No demanda servicios sociales $265(89.8 \%)$ y mantiene contacto con servicios sociales 91 (30.8\%). Solamente demandan algún tipo de ayuda 15 (5.1\%).

Reconocen el problema $50(16.9 \%)$ y justifican la acumulación $26(8.8 \%)$. Entre los casos, $283(95.6 \%)$ son de nacionalidad española (tablas 1 y 2).

Tabla1.

Características sociodemográficas de los acumuladores patológicos de la ciudad de Madrid, 2009-2012.

\begin{tabular}{|c|c|c|}
\hline VARIABLE & $\mathbf{N}$ & $\begin{array}{l}\text { Frecuencias absolutas y relativas } \\
\text { con criterios válidos. }\end{array}$ \\
\hline $\begin{array}{l}\text { EDAD (en años) } \\
\text { Media } \\
\text { DS } \\
\text { Rango } \\
\text { Distribución por grupos } \\
<45 \\
\text { DE } 46 \text { a } 65 \\
>65 \\
\text { Desconocido }\end{array}$ & 295 & $\begin{array}{l}64.77 \\
15 \\
23-96 \\
\\
29(9.8) \\
118(40) \\
139(47.1) \\
9(3.4)\end{array}$ \\
\hline
\end{tabular}


(continúa Tabla 1)

\begin{tabular}{|c|c|c|}
\hline $\begin{array}{l}\text { SEXO } \\
\text { Mujer } \\
\text { Hombre }\end{array}$ & 295 & $\begin{array}{l}130(44.1) \\
165(55.9)\end{array}$ \\
\hline NACIONALIDAD & 295 & \\
\hline Española & & $283(95.9)$ \\
\hline Extranjero & & $9(3.1)$ \\
\hline No especificado & & $3(1)$ \\
\hline NUEVA & 295 & $250(84.7)$ \\
\hline REINCIDENTE & & $45(15.3)$ \\
\hline NIVEL DE ESTUDIOS & 245 & \\
\hline Analfabeto & & $46(18.8)$ \\
\hline Leer/escribir & & $79(32.2)$ \\
\hline Primarios & & $50(20.4)$ \\
\hline Secundarios & & $64(26.1)$ \\
\hline Sin Especificar & & $6(2.4)$ \\
\hline SITUACIÓN LABORAL & 229 & \\
\hline Activo & & $39(17.0)$ \\
\hline Parado & & $40(17.9)$ \\
\hline Pensionista & & $149(65.1)$ \\
\hline FAMILIARES & 294 & \\
\hline Sí & & $42(14.3)$ \\
\hline No & & $252(85.7)$ \\
\hline RELACIÓN CON FAMILIA & 258 & \\
\hline Buena & & $62(24.0)$ \\
\hline Mala & & $196(76.0)$ \\
\hline VIVE SOLO & 295 & \\
\hline Sí & & $132(44.7)$ \\
\hline No & & $163(55.3)$ \\
\hline AISLAMIENTO SOCIAL & 294 & \\
\hline $\mathrm{Si}$ & & $32(10.9)$ \\
\hline No & & $262(89.1)$ \\
\hline SOCIABLE & 295 & \\
\hline Sí & & $91(30.8)$ \\
\hline No & & $204(69.28)$ \\
\hline RESERVADO & 295 & \\
\hline $\mathrm{Si}$ & & $56(19.0)$ \\
\hline No & & $239(81.0)$ \\
\hline AGRESIVO & 295 & \\
\hline Sí & & $23(7.8)$ \\
\hline No & & $272(92.2)$ \\
\hline DEMANDA SERVICIOS SOCIALES & 295 & \\
\hline & & $30(10.2)$ \\
\hline No & & $265(89.8)$ \\
\hline MANTIENE CONTACTO CON SERVICIOS SOCIALES & 295 & \\
\hline & & $91(30.8)$ \\
\hline No & & $204(69.2)$ \\
\hline DEMANDA AYUDA & 295 & \\
\hline Sí & & $15(5.1)$ \\
\hline No & & $280(94.9)$ \\
\hline RECONOCE PROBLEMA & 295 & \\
\hline Sí & & $50(16.9)$ \\
\hline No & & $245(83.1)$ \\
\hline \begin{tabular}{|l|l} 
JUSTIFICA ACÚMULO \\
\end{tabular} & 295 & \\
\hline Sí & & $26(8.8)$ \\
\hline No & & $269(91.2)$ \\
\hline
\end{tabular}


Tabla 2.

Sexo, edad, edad media y desviación típica

\begin{tabular}{|l|l|l|l|l|l|l|}
\hline & N: 286(\%) & $<$ DE 45 AÑOS (\%) & $\begin{array}{l}\text { DE 46 A 65 AÑOS } \\
(\%)\end{array}$ & DE 65 AÑOS (\%) & MEDIA & $\begin{array}{l}\text { DESVIACIÓN } \\
\text { TÍPICA }\end{array}$ \\
\hline HOMBRE & $159(55.7)$ & $20(12,6)$ & $68(42,8)$ & $71(44,7)$ & 62.79 & 15.048 \\
\hline MUJER & $127(44.3)$ & $9(7,1)$ & $50(39,4)$ & $68(53,5)$ & 67.24 & 15.115 \\
\hline
\end{tabular}

Por distritos destacan San Blas con 4.86 y Puente de Vallecas con 4.06 casos por cada 10.000 viviendas respectivamente (figura 1).

Los que viven solos son los más acumuladores, 85 (73,3\%) (figura 2).

Características de la vivienda y del acúmulo; presencia de animales.

En 273 (92.5\%) el lugar de acumulación de enseres y/o basura es un piso o apartamento, ocupando toda la vivienda en $171(68.1 \%)$. La tipología que con más frecuencia se acumula es inorgánica, 140 (55.1\%).

En $53(18.0 \%)$ casos hay presencia de animales (perro, gato, aves), la mayoría, 31 (58.5\%) en malas condiciones higiénico-sanitarias, serían los casos asimilables al llamado Síndrome de Noé (figura 3); existe presencia de artrópodos y/o roedores en $75(25.4 \%)$.

En 129 (43.6\%) se retiraron $260.346 \mathrm{Kgs}$. (mediana $1220 \mathrm{kgs}$, rango intercuartílico $2240 \mathrm{Kgs}$ ) (Tabla 3).

Figura 1.

Localización de los casos de trastorno por acumulación en los distritos de madrid de 2009 a 2012. Casos por cada 10000 hogares.

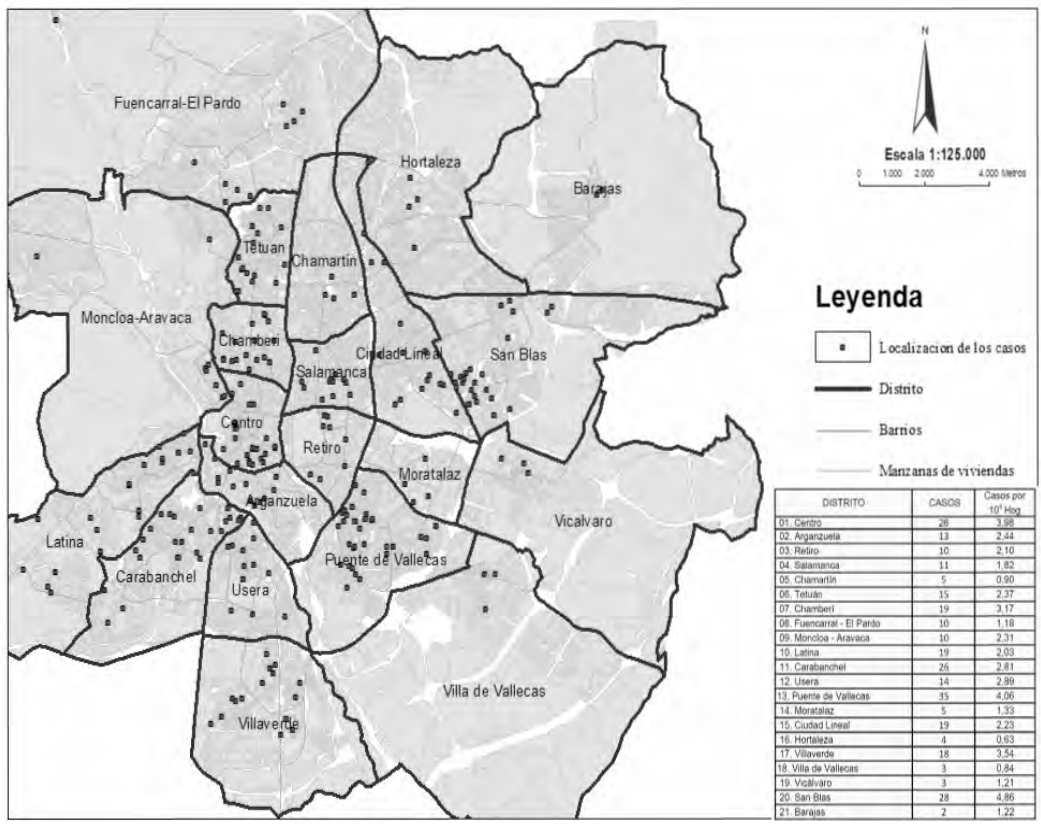


Figura 2.

Relación vivir solo con distribución del acúmulo.

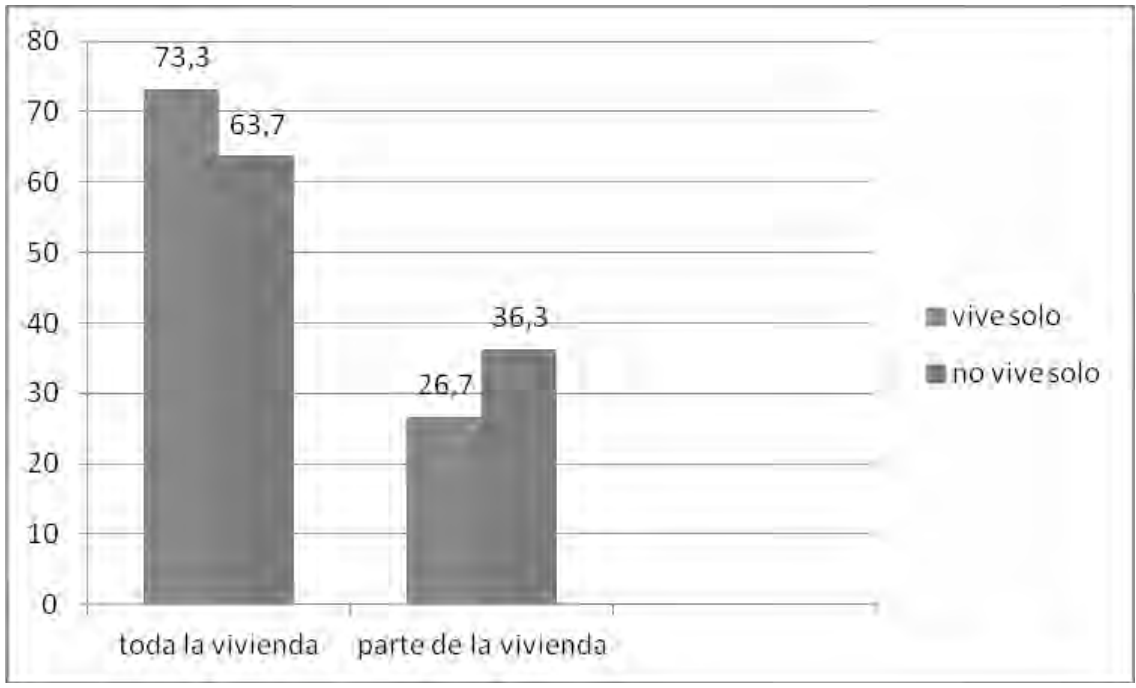

Figura 3.

Relación Sexo/Edad/Tipología del acúmulo en \%. N: 247.

\begin{tabular}{|c|c|c|c|c|c|c|c|}
\hline \multicolumn{7}{|l|}{70} & \multirow{12}{*}{$\begin{array}{l}\text { INORGÁNICO } \\
\text { ORGANICO } \\
\text { MIXTO }\end{array}$} \\
\hline 60 & & & & & & & \\
\hline 50 & & & & & & & \\
\hline 40 & & & & & & & \\
\hline 30 & & & & & & & \\
\hline 20 & & & & & & & \\
\hline $10-$ & & & & & & & \\
\hline 0 & $<45$ & $45-65$ & $>65$ & $<45$ & $45-65$ & $>65$ & \\
\hline & & HOMBR & & & MUJER & & \\
\hline INORGÁNICO & 50 & 57,9 & 58,7 & 14,3 & 55,6 & 49,1 & \\
\hline ORGANICO & 5,6 & 3,5 & 1,6 & 28,6 & 6,7 & 1,8 & \\
\hline MIXTO & 44,4 & 38,6 & 39,7 & 57,1 & 37,8 & 49,1 & \\
\hline
\end{tabular}


Tabla 3.

Características del la vivienda y del acúmulo.

\begin{tabular}{|c|c|c|}
\hline VARIABLE & $\mathbf{N}$ & $\begin{array}{l}\text { FRECUENCIAS ABSOLUTAS(\%). } \\
\text { VÁLIDO }\end{array}$ \\
\hline $\begin{array}{l}\text { LUGAR DEL ACÚMULO } \\
\text { Piso/apartamento } \\
\text { Vivienda unifamiliar } \\
\text { Patio } \\
\text { Infravivienda } \\
\text { Local } \\
\end{array}$ & 295 & $\begin{array}{l}273(92.5) \\
6(2.0) \\
82.7) \\
3(1.0) \\
5(1.7) \\
\end{array}$ \\
\hline $\begin{array}{l}\text { TIPOLOGIA DEL ACÚMULO } \\
\text { Inorgánico } \\
\text { Orgánico } \\
\text { Mixto }\end{array}$ & 254 & $\begin{array}{l}140(55.1) \\
10(3.9) \\
104(40.9)\end{array}$ \\
\hline $\begin{array}{l}\text { DISTRIBUCIÓN DEL ACÚMULO } \\
\text { Toda la vivienda } \\
\text { Parte de la vivienda }\end{array}$ & 251 & $\begin{array}{l}171(68.1) \\
80(31.9)\end{array}$ \\
\hline $\begin{array}{l}\text { KR. RETIRADOS. } \\
\text { Total } \\
\text { Mediana } \\
\text { Rango Intercuartílico } \\
\text { Rango }\end{array}$ & 129 & $\begin{array}{l}260.346 \\
2107 \\
2018 \\
30-11400\end{array}$ \\
\hline $\begin{array}{l}\text { PRESENCIA DE PERRROS, GATOS, AVES } \\
\text { Sí } \\
\text { No }\end{array}$ & 295 & \begin{tabular}{|l}
$53(18.0)$ \\
$242(82.0)$
\end{tabular} \\
\hline $\begin{array}{l}\text { SITUACIÓN HIGIÉNICO-SANITARIA DE LOS ANIMALES } \\
\text { Buenas condiciones } \\
\text { Malas condiciones }\end{array}$ & 53 & $\begin{array}{l}22(41.5) \\
31(58.5)\end{array}$ \\
\hline $\begin{array}{l}\text { ARTRÓPODOS, ROEDORES } \\
\text { SÍ } \\
\text { NO } \\
\end{array}$ & 295 & $\begin{array}{l}75(25.4) \\
220(74.6)\end{array}$ \\
\hline
\end{tabular}

Si relacionamos el sexo, la edad y las características de lo acumulado, los hombres de más de 65 años acumulan con más frecuencia materia inorgánica 37 $(58,7 \%)$; no se aprecia una relación lineal entre la edad y el acúmulo de diferentes tipologías, aunque en la tipología orgánica si parece tender a concentrarse en los acumuladores más jóvenes, tanto en hombres como en mujeres.

\section{Saneamiento y recidivas}

Los que tienen entre 45-65 años son los más reincidentes 25 (21.2\%). Resulta más probable que reincidan los casos en los que se ha realizado el saneamiento mediante autorización judicial de entrada en domicilio 15 (20.8\%) (Tabla 4).

Tabla 4.

Forma de saneamiento y edad de los casos nuevos y reincidentes.

\begin{tabular}{|l|l|l|}
\hline N: 286 & NUEVO (\%) & REINCIDENTE (\%) \\
\hline EDAD(en años) & & \\
\hline$<45$ & $24(82.8)$ & $5(17.2)$ \\
$45-65$ & $93(78.8)$ & $25(21.2)$ \\
$>65$ & $124(89.2)$ & $15(10.8)$ \\
\hline SANEAMIENTO & & \\
VOLUNTARIA & $72(91.1)$ & $7(8.9)$ \\
JUDICIAL & $57(79.2)$ & $15(20.8)$ \\
INQUILINO & $66(85.7)$ & $11(14.3)$ \\
EN BLANCO & $54(81.2)$ & $12(18.2)$ \\
\hline
\end{tabular}




\section{Patología asociada.}

De los hombres que acumulan son alcohólicos 20 (31.7\%). Las mujeres que presentan el trastorno por acumulación están diagnosticadas de Depresión 29 $(59.2 \%)$ (Tabla 5).

Tabla 5.

Sexo y comorbilidad

\begin{tabular}{|c|c|c|c|c|c|c|c|c|}
\hline & \multicolumn{4}{|c|}{$\begin{array}{l}\text { ADICCIONES(N:107) } \\
\text { N (\%) }\end{array}$} & \multicolumn{4}{|c|}{$\begin{array}{l}\text { PATOLOGÍAS(N:113) } \\
\text { N (\%) }\end{array}$} \\
\hline & $\begin{array}{l}\text { ALCOHÓ } \\
\text { LICO }\end{array}$ & $\begin{array}{l}\text { TOXICÓ- } \\
\text { MANO }\end{array}$ & OTROS & NO & $\begin{array}{l}\text { ALZHEIMER } \\
\text { y/o DEMENCIA }\end{array}$ & $\begin{array}{l}\text { DISMINUIDO } \\
\text { PSÍQUICO } \\
\text { OTRAS }\end{array}$ & DEPRESIÓN & $\begin{array}{l}\text { DISMINUIDO } \\
\text { FíSICO }\end{array}$ \\
\hline HOMBRE & $20(31,7)$ & $3(4,8)$ & $1(1,6)$ & $39(61,9)$ & $3(6.9)$ & $20(34,5)$ & $28(48,3)$ & $6(10,3)$ \\
\hline MUJER & $5(10,0)$ & $3(6,0)$ & $1(2,0)$ & $41(82,0)$ & $6(12,2)$ & $12(24,5)$ & $29(59,2)$ & $2(4,1)$ \\
\hline
\end{tabular}

Los que presentan algún tipo de patología acumulan más inorgánico 57 (60\%). Los clasificados como disminuidos psíquicos $21(70.0 \%)$ son los que más acumulan y lo hacen acumulando materia inorgánica. Solo son acumuladores de materia orgánica los que están diagnosticados de depresión 4 (8\%) (Tabla 6).

Tabla 6.

Patología y tipología del acúmulo

\begin{tabular}{|l|l|l|l|}
\hline N. 95 & TIPOLOGIA DEL ACÚMULO \\
\hline PATOLOGIA & INORGÁNICO & ORGÁNICO & MIXTO \\
\hline DISMINUIDO PSIQQUICO OTRAS & $21(70,0)$ & 0 & $9(30,0)$ \\
\hline DEMENCIA Y/O ALZHEIMER & $4(44.5)$ & 0 & $5(55.5)$ \\
\hline DEPRESIÓN & $30(60,0)$ & 4 & $16(32,0)$ \\
\hline DISMINUIDO FISICO & $2(33,3)$ & 0 & $4(66,7)$ \\
\hline Total & $57(60.6 \%)$ & $4(4.2 \%)$ & $34(35.8 \%)$ \\
\hline
\end{tabular}

\section{Discusión:}

El objetivo principal de nuestro estudio es conocer las características sociodemográficas de las personas que presentan un trastorno por acumulación en la ciudad de Madrid. Las personas que sufren este trastorno reúnen una serie de características tales como no reconocer el problema, vivir solos, comportamiento insociable, demandar muy rara vez algún tipo de ayuda y ser muy pocos conscientes de su trastorno a pesar de no presentar ningún déficit cognitivo significativo $(19,25-$ 28). Este comportamiento dificulta su accesibilidad y estudio y originan un retraso diagnóstico que impide la intervención precoz y eficaz (29) y obligan a realizar su estudio fuera de la clínica (31). Estas circunstancias explican el elevado número de valores ausentes en muchas de las variables evaluadas. 
Lo novedoso de nuestro estudio radica en la procedencia o forma de obtener los casos; la selección de los acumuladores se ha hecho a través de denuncias motivadas por las consecuencias físicas o sociales del comportamiento del sujeto en el entorno personal, familiar y vecinal, no mediante escalas o test estandarizados empleados en la literatura para hacer diagnósticos clínicos. Se trata de una aproximación clínica a través de una ruta administrativa. Esta, por decirlo de alguna manera, vía indirecta de entrada a través de denuncias ofrece la posibilidad de identificar a los acumuladores, algo que de otra manera sería imposible.

Este estudio es el primero en examinar las características sociodemográficas de una población de acumuladores patológicos obtenida partir de denuncias de posibles casos de insalubridad ante cualquier organismo o institución pública en el Municipio de Madrid.

Distinguir entre los diferentes tipos de conducta acumuladora (8) va más allá de las pretensiones del presente estudio.

Coincidiendo con lo encontrado por otros estudios $(15,18,23)$, la prevalencia aumenta con la edad y es más elevada en los hombres. Al no tratarse de una muestra significativa de la población de acumuladores no podemos afirmar que los hombres son más acumuladores que las mujeres.

Hay autores que fijan la edad de inicio de la conducta acumuladora en la adolescencia $(15,23,29,31-33)$. Otros, sin embargo, reportan que las edades medias serían la edad de inicio y sería la gravedad lo que se incrementa con la edad. Se trataría, pues, de una condición crónica y progresiva (32); a día de hoy no sabemos si se trata de un incremento en el número de casos según se envejece o a un incremento de la severidad con los años (25).

Dado que la significación clínica del acumulador es progresiva, a nosotros nos llegan los casos más severos o avanzados, cuando ya originan problemas de Salud Pública, de seguridad y de convivencia. Conocer el inicio de la conducta acumuladora excede la pretensión de este estudio por lo que poco podemos añadir a los posibles factores causales.

Los casos reincidentes se dan con más frecuencia de 45-65 años, a diferencia de otros estudios que refieren que este comportamiento se manifiesta en edades más jóvenes (32).

Los hombres y las mujeres mayores de 65 años acumulan con más frecuencia tipología inorgánica y mixta. Cuando se trata de materia orgánica son los hombres y las mujeres más jóvenes, entre los 45 y los 65 años, aunque resulta un dato poco valorable dado el limitado número de casos.

En los Distritos de San Blas y Puente de Vallecas se reportan más casos, coincidente con otros estudios que señalan este fenómeno como más prevalente en las zonas más caóticas o de menos ingresos (34). 
El reducido número de casos entre extranjeros contrasta con la población promedio de dicha población en esos años en la ciudad de Madrid, 17\% (543.830) (34), pero se mantiene la proporción de expedientes abiertos a extranjeros $4.27 \%$ (49/1147) y los que tienen comportamiento acumulador 3.1\% (9/295). La posibilidad de que existiera una vertiente xenófoba en el número de denuncias queda, en cierta forma, desmentida con estos datos.

En contradicción con otros estudios (4), son más frecuentes los casos cuando conviven con otras personas en relación con los que viven solos.

En nuestro estudio los hombres que viven solos son los que más acumulan (56.8\%) aproximándose a lo descrito por otros que refieren que los nunca casados tienen síntomas más severos (27); tal vez por tener mayor apego a las cosas por no tener pareja, o que el conviviente frena la conducta acumuladora.

El trastorno por acumulación se da con más frecuencia, en nuestro estudio, entre las pensionistas, en otros se señala a los que no tienen trabajo (20). Probablemente nos estemos refiriendo a la misma población dada la forma en que hemos descrito nosotros esta variable.

Como describen la mayoría de los estudios y el nuestro, la conducta acumuladora ocurre de forma más frecuente en ausencia de trastornos mentales. En algunos estudios se describe hasta un $44 \%$ de acumuladores con alguna enfermedad mental (27).

La depresión es la patología acompañante más frecuente en nuestra serie, como señalan otros autores $(11-12,19,23-24,35)$. La alta frecuencia de esta condición nos plantea preguntas acerca del rol de la misma en la génesis del trastorno por acumulación. ¿Resulta una patología previa y empeora la conducta acumuladora o es a la inversa?

El alcohol podría interferir en cómo se organiza la vida el acumulador y le incapacita para poder desechar las cosas inservibles (15).

Debido a que el registro de los trastornos mentales no está basada en clínicos con experiencia ni en métodos adecuados de evaluación, se necesita estandarizar la evaluación mental y físicas para determinar si el comportamiento acumulador está relacionado con patología mental o biológica. Algunos estudios señalan que alguna personas acumuladoras inician su conducta después de una lesión cerebral que afecte a la zona prefrontal ventromedial del córtex $(11,36)$.

Fue necesaria la autorización judicial de entrada en domicilio en 72 casos de los que sólo reincidieron un $20.8 \%$. Este dato parece apuntar en sentido contrario con los que afirman que la limpieza involuntaria $(4,23,25,37)$,contra la voluntad del acumulador, no supone una solución a este problema, pero tal vez sí una atenuación.

$\mathrm{Al}$ ser un trastorno tan poco frecuente resulta muy difícil y costoso conocer la prevalencia en la población general (27). Si extrapolamos la cifra de prevalencia del $0.4 \%$ (15) al 2-5\% de algunos estudios $(18-19,21)$, significaría que en la ciudad de Madrid habría aproximadamente entre 10.762 y 134.534 personas con conducta acumuladora(34), cifra que nos parece claramente desproporcionada en relación 
con los datos que apuntan nuestro estudio y otros(el cálculo se ha realizado promediando la población de Madrid mayor de 19 años en los 4 años del estudio).

Una de las limitaciones del estudio viene motiva por desconocer si nuestra población es representativa de la población de acumuladores. Sin embargo, la centralización en la resolución administrativa de este complejo problema de insalubridad en el Ayuntamiento de Madrid, junto el elevado número de casos de nuestra serie, la convierte en una aproximación bastante fiel a la población de acumuladores patológicos del Municipio de Madrid y a toda la población que presenta este trastorno de conducta. Gracias a la posición estratégica que ocupa la Unidad Técnica de Entorno Urbano y Vivienda en la resolución de estos casos en toda la ciudad, solo se escaparían los casos leves o incipientes, los que de forma exclusiva acudan a los servicios sanitarios y aquellos a los que se enfrente de forma autónoma la familia; de estas tres vías, la mayoría de los casos finalmente se añaden a nuestra casuística dado que el difícil manejo de los mismos precisa de la intervención de los Servicios Sociales Municipales que sistemáticamente los derivan a nuestra Unidad.

El registro de los datos a partir de la observación directa y la entrevista por parte de los inspectores añade variabilidad a los mismos. A pesar de ello creemos que una de las fortalezas de nuestro estudio radica en que los datos son recogidos y registrados por tres inspectores experimentados, siempre los mismos, en una ficha estructurada, y que con frecuencia realizan las inspecciones de forma conjunta, lo que facilita la homogeneidad en el registro y en el contenido de la información.

Por todo ello pensamos que nuestros resultados contribuyen a incrementar el conocimiento de las personas que presentan trastornos por acumulación.

Se requieren de futuros estudios epidemiológicos, clínicos y de laboratorio (12) en la población general y en grupos seleccionados que nos permitan conocer la prevalencia y la historia natural de este problema de conducta. Estos conocimientos permitirían acercarse al verdadero objetivo de mejorar la función y los problemas de salud de las personas con conducta acumuladora que facilite la permanencia en el domicilio y en su entorno familiar y vecinal, reduciendo el sufrimiento del individuo y el elevado coste económico que provoca en la sociedad. A todos los usuarios en este estudio se les ofreció la posibilidad de recibir atención por parte de Servicios Sociales Comunitarios pero en la mayoría de los casos se rechazó pese a la aparente necesidad de estos servicios por su parte.

Los acumuladores resultan un desafío ético y legal, relacionado con la capacidad del individuo, la seguridad personal y de la comunidad. Uno de los grandes dilemas es clarificar la capacidad del acumulador, para cuidarse, manejar las finanzas personales y tomar decisiones que permitan aclarar si puede vivir en un entorno considerado peligroso para él y su entorno. 
La ausencia de autocuidados, las caídas en personas mayores y alguno trastornos mentales pueden ser signos gatillo que despierten la sospecha de los médicos de Atención Primaria y los profesionales de Salud Mental.

La necesidad de realizar un abordaje integral del problema con la implicación fundamentalmente de los servicios de Salud Mental, Servicios Sociales Comunitarios y en muchos casos la Judicatura sería una de las lecturas de nuestro trabajo. Como todos los problemas de salud complejos su manejo debe hacerse desde una aproximación multidisciplinar.

BIBLIOGRAFÍA:

(1) Frost RO, Gross RC. The Hoarding of Possessions. Behav Res.Ther. (1993).31(4): 367381.

(2) Pertusa A., et al.Refining the diagnostic boundaries of compulsive hoarding: A critical review. Clin. Psychol. (Rev. 2010), doi: 10.1016/j.cpr.2010.01.007.

(3) Snowdon J, Shah A, Halliday G. Severe domestic squalor: a review. Int Psychogeriatr. (2007) Feb; 19(1):37-51.

(4) Frost RO, Hartl TL. A cognitive-behavioral model of compulsive hoarding. Behav. Res. The. (1996); vol. 34, (4): 341-350.

(5) Frost RO, Steketee GS. Clinical Aspects and Treatement Strategies. En: Jenike MA, Baer L, Minichiello WE, Ed.D editors. Obsessive-Compulsive Disorders. Practical Management. Chapter 23. Third Edition. 1998.

(6) Mataix-Cols D, Frost RO, Pertusa A, Clark LA, Saxena S, Leckman JF et al. Hoarding Disorder: Anew diagnosis for DSM-V?. Depress Anxiety. (2010); 27:556-572.

(7) Lahera, G; Saiz-González, D; Martín-Ballesteros, E; Pérez-Rodríguez, M. M; BacaGarcía, Enrique. Diagnóstico diferencial de la conducta acumuladora. Actas esp. Psiquiatr. (2006); 34(6):403-407, Nov-dic.

(8) Gómez-Feria PI. Formas clínicas de las conductas acumuladoras. Psiq Biol. (2009); 16(3):122-30.

(9) Becerra GJA, Robles JMJ. Características del trastorno por acumulación. ¿Un nuevo síndrome clínico? Psiq Biol. (2010); 17(3):111-113.

(10) Carrato VE, Martínez AR. Síndrome de Diógenes. A propósito de un caso. Rev. Asoc. Esp. Neuropsip, (2010); 30(107), 489-495.

(11) Saxena S. Recent advances in compulsive hoarding. Curr Psychiatry Rep (2008), 10: 297-303.

(12) Tolin DF, Villavicencio A, Umbach A, Kurtz MM; Neuropsychological Functioning in Hoarding Disorder; (2011), 189(3): 413-418.

(13) Grisham JR, Norberg MM. Compulsive hoarding: current controversies and new directions. Dialogues Clin Neurosci. (2010). vol 12(2): 233-240.

(14) Frost RO, Kim HJ, Morris C, Bloss C, Murray-Close M, Steketee G. Hoarding, compulsive buying and reasons for saving. Behav Res Ther; (1998) 36; 657-664. 
(15) Frost R O, Steketee G, Grisham J. Measurement of compulsive hoarding: saving inventory-revised. Behav Res Ther. (2004); 42: 1163-1182.

(16) Caballero GJC. Síndrome de Diógenes. An Psiquiatria.(1992); 8:21-26.

(17) Jiménez JE, Molina FN, Díaz PF, Reyes TA. El mal llamado "Síndrome de Diógenes". Enfermeria Docente. (2008); 88: 36-39.

(18) Samuels JF, Bienvenu OJ, Grados MA, Cullen B, Riddle MA, Liang K et al. Prevalence and Correlates of Hoarding Behavior in a Community-Based Sample. (2008) 46, (7), 836-844.

(19) Frost RO, Steketee G, Tolin DF; Comorbidity in Hoarding Disorder. Depress Anxiety. (2011); 28(10): 876-884.

(20) Saxena S, Ayers CR, Maidment KM, Vapnik T, Wetherell JL, Bystritsky A; Quality of Life and Functional Impairment in Compulsive Hoarding; J Psychiatr Res. (2011); 45(4): 475-480.

(21) Beyond overwhelmed. San Francisco Task Force on Compulsive Hoarding. 2009.

(22) Patronek GJ. Hoarding of animals: An Under-Recognized Public Health Problem in a Difficult-to-study Population. Public Health Rep. (1999) ; 14: 81-87.

(23) Frank C, Misiaszek B. Approach to hoarding in family medicine: beyond reality television. Can Fam Physician. (2012) Oct; 58(10): 1087-91.

(24) Tolin DF, Frost RO, Steketee G, A brief interview for assessing compulsive hoarding: The hoarding rating Scale-Interview. Psychiatry Res. (2010); 178(1): 147-152.

(25) Steketee Gl; Frost RO, Kim HJ. Hoarding by elderly people. Health Soc Work. (2001) 26 (3): 176-184.

(26) Samuels J, Bienvenu III OJ, Riddle MA, Cullen BAM, Grados MA., Liang KY. Hoarding in obsessive-compulsive disorder: results from a case-control study. Behav Res Ther. (2002); 4: 517-528.

(27) Seedat S, Stein DJ. Hoerding in obsessive-compulsive disorder and related disorder. A preliminary report of 15 cases. Psychiatry and Clinical Neuroscience. (2002). 56:17-23

(28) Tolin DF. Understanding and treating hoarding. A biopsycosocial perspective. J Clin Psychol. (2011); 67: 517-526.

(29) Frost RO, Tolin DF, Steketee G, Fitch KE, Selbo-Bruns A; Excessive Acquisition in Hoarding. J Anxiety Disord. (2009). 23(5): 632-639.

(30) Frost RO, Steketee G, Williams L. Hoarding: a community health problem. (2000). Health Soc Care Community. (2000). (4): 229-234.

(31) Storch EA, Lack CW, Merlo LJ, Geffken GR, Jacob ML, Murphy TK. Clinical features of children and adolescents with obsessive-compulsive disorder and hoarding symptoms. Compr Psychiatry. (2007); (48): 313-318.

(32) Ayers Cr, Saxena S, Golshan S, Wetherell JL. Age at onset and clinical features of late life compulsive hoarding. Int J of Geriatric Psyquiatry. (2009); 23(2):142-149.

(33) Sansone RA, Sansone LA; Hoarding: Obsessive symptom or Syndrome; Psychiatry (Edgemont); (2010); 7(2): 24-27.

(34) Explotacion del Padron Municipal de Habitantes 2012. Areas de información Estadística. Estadística Ayuntamiento de Madrid. Disponible en http://www.madrid.es/portales/munimadrid/ es/Inicio/Ayuntamiento/Estadistica/Areas-de-informacion-estadistica/Economia/Renta. (consultado el 13/12/2013).

(35) Halliday G, Banerjee S, Philpot M ; Macdonald A. Community study of people who live in squalor. The Lancet. (2000); (355): $882-886$. 
ORIGINALES Y REVISIONES

(36) Tolin DF, Stevens MC, Villavicencio A, Norberg M, Calhoun VD, Frost RO et al; Neural Mechanisms of Decisión Making in Hoarding Disorder. Arch gen Psychiatry;( 2012); 69(8): 832-841.

(37) Sorrell JM. Understanding hoarding in older adults. J Psychosoc Nurs Ment Health Serv. (2012); 50(3):17-21. 